\title{
Feasibility of Cell Planning for the L-Band Digital Aeronautical Communications System under the Constraint of Secondary Spectrum Usage
}

\author{
Mohamad Mostafa, Miguel A. Bellido-Manganell, and Thomas Gräupl
}

\begin{abstract}
The L-band digital aeronautical communications system (LDACS) is a cellular air-ground data link for air traffic control. It is a corner-stone of the future aeronautical communications infrastructure. LDACS shall operate in the frequency band 960-1164 MHz under the constraint of secondary spectrum usage. This implies strict spectral, power, and spatial separation towards current legacy systems operating in this frequency band. It has been proposed to fulfil these restrictions using cell planning for LDACS without changing frequency allocations of the legacy systems. However, the feasibility of such a cell planning has yet to be demonstrated. In this paper we demonstrate the feasibility of LDACS cell planning under these constraints. For this purpose we introduce the theoretical limits for such a separation enabling the co-existence of LDACS and DME (distance measuring equipment system: the primary user) in the same frequency band. Closed-form expressions are obtained such that the proper operation of DME is not harmfully affected by LDACS. These expressions are utilized in the first step to find DME-compliant locations for LDACS ground stations. In the second step, interference constraints within LDACS itself are defined and applied. This approach yields DME-compliant locations of LDACS ground stations with channel assignments fulfilling the interference constraints. The application of our method shows that LDACS cell planning in Europe is possible without disturbing the proper operation of the DME system.
\end{abstract}

Index Terms-L-band digital aeronautical communications system (LDACS), distance measuring equipment (DME), cell planning, channel assignment, Hopfield networks.

\section{INTRODUCTION}

The frequency band $960-1164 \mathrm{MHz}$ is reserved worldwide for the aeronautical radio navigation service (ARNS). However, considering that the frequency band $117.975-137 \mathrm{MHz}$, currently allocated for the aeronautical mobile (route) service $(\mathrm{AM}(\mathrm{R}) \mathrm{S})$, will not be able to support data communications in certain areas in the medium and long term [1] and that the utilization factor of the frequency range $960-1525 \mathrm{MHz}$ is only about 0.0236 [2], the World Radiocommunication Conference [3] [4] allocated the frequency band 960-1164 $\mathrm{MHz}$ for $\mathrm{AM}(\mathrm{R}) \mathrm{S}$ systems under the constraint that existing aeronautical radio navigation services are not harmfully interfered. This allocation shall enable the operation of new data intensive applications and concepts currently introduced by

Copyright (c) 2015 IEEE. Personal use of this material is permitted However, permission to use this material for any other purposes must be obtained from the IEEE by sending a request to pubs-permissions@ieee.org.

Institute of Communications and Navigation, German Aerospace Center (DLR), Münchener Straße 20, 82234 Weßling, Germany. E-mail \{Mohamad.Mostafa, Miguel.BellidoManganell, Thomas.Graeupl\}@DLR.de
SESAR [5] and NextGen [6] for a resilient modernization of air traffic management (ATM).

Jointly developed by Eurocontrol and the Federal Aviation Administration (FAA) for air traffic management in civil aviation [7], the future communications infrastructure (FCI) foresees a set of data links integrated into a single communications network. The L-band digital aeronautical communications system (LDACS) [8] is envisaged as the air-ground communications component within the FCI. LDACS is a frequencydivision duplex communications system utilizing orthogonal frequency-division multiplexing (OFDM) with adaptive coding and modulation, $500 \mathrm{kHz}$ effective bandwidth, reservationbased access control, and advanced network protocols [9] [10].

LDACS as an $\mathrm{AM}(\mathrm{R}) \mathrm{S}$ is intended to operate in the frequency band 960-1164 MHz. However, the distance measuring equipment (DME), an aeronautical radio navigation system, is the primary user of this frequency band. Therefore, the physical layer design of LDACS has to assure the co-existence with the DME system. This is to be achieved by: (i) minimizing the out-of-band radiation towards DME devices [11], and (ii) implementing at the LDACS receiver the required interference robustness against interference from the DME system [12] [13]. In addition, the frequencies to be used by LDACS must be chosen in such a way that harmful interference between LDACS and DME is avoided. The common approach followed by most radio systems is to exclusively use a frequency band where no other system is allowed to transmit. This way, cell planning has only to take into account interference within the system itself. Nevertheless, given the scarce vacant spectrum available in the L-band, this approach is not feasible for LDACS. Consequently, shared use of the spectrum allocated for DME is necessary. However, the feasibility of an LDACS cell planning under spectrum sharing conditions with the DME system, where the proper operation of DME is not affected by the operation of LDACS, is still to be shown. Spectrum sharing can be achieved by accessing the spectrum dynamically and/or by following a hierarchical access model, where a secondary user, i.e., LDACS, is allowed to access a licensed frequency band as long as no harmful interference is caused to the primary user, i.e., DME. Such a shared access to the spectrum would not only allow LDACS to operate in the Lband, but is also a promising technique to improve spectrum utilization [14]. Three approaches for spectrum sharing are commonly proposed: underlay, interweave, and overlay.

In the underlay approach, the power of the secondary signals at a primary receiver lies below the noise floor of the primary 
receiver. Such an approach enables the simultaneous access to the licensed frequency band by primary and secondary users but imposes a substantial restriction on the transmit power of secondary transmitters and is only suitable for short range communications [15]. Taking the transmit power of LDACS transmitters and the possible cell sizes of LDACS [8] into account we conclude that the underlay approach is not suitable for LDACS in the foreseen frequency band.

In the interweave approach, secondary users access the licensed frequency band in a way orthogonal to the primary user in the signal space [14]. Therefore, the interweave approach enables an opportunistic utilization of the licensed frequency band by secondary users in the temporal and spatial domain where the licensed frequency band is not occupied by the primary users. Based on the density of the DME network on ground and the fact that DME onboard devices are mobile and have a high radio visibility, it is unlikely that an LDACS cell planning with a complete coverage is possible based on the spatial dynamic of the licensed frequency band. Moreover, LDACS cell planning based on the temporal dynamic of the licensed frequency band is unsuitable since the latency requirements of LDACS cannot be guaranteed in this case.

Finally, in the overlay approach, secondary transmitters are allowed to access the licensed frequency band as long as the interference constraints at all primary receivers are fulfilled. For this purpose, interference temperature has been introduced in [16]. The interference temperature limit represents the maximum amount of interference that a receiver can tolerate [17].

In this paper, we adopt the overlay approach to show the feasibility of a pan-European LDACS cell planning fulfilling interference constraints towards the DME system. This approach is followed because (i) the interference temperature limit of DME devices has been obtained in [18] as the maximum LDACS interference that a DME device can receive without affecting its proper operation, and (ii) worst-case interference conditions towards the DME system can be calculated by using the publicly available geolocation database of the DME platforms. The DME-compliant LDACS cell planning presented in this paper is performed in two steps:

- Step 1: Finding a set of locations for LDACS ground stations (LDACS GSs) equipped with specific transmit channels (i.e., frequencies for transmission) and transmit power such that the proper operation of DME devices is not affected.

- Step 2: Ensuring that interference constraints within LDACS itself are fulfilled. We call the second step the channel assignment task.

The interference temperature limit of DME devices is used in the first step to decide which locations can be used for LDACS GSs without affecting the proper operation of the DME devices. To this end, the geolocation database of the primary receivers (DME devices) together with the essential parameters of LDACS transmitters, and worst-case geometrical arrangement between primary and secondary users are used to estimate the power of the transmitted secondary signal at the primary receiver. Following this, the channel assignment task is modeled as finding the maximum independent set in a graph, a clique in the graph's complement [19], which is generally an NP-complete problem [20]. Two-state Hopfield neural networks are employed to find a suboptimum solution for this problem because of their guaranteed and fast convergence [21]. For this, we follow a similar approach as in [21], and restrict the DME-compliant LDACS cell planning to the forward link (FL: from ground station to airborne station). However, a suitable frequency band for the reverse link (RL: from airborne station to ground station) is suggested, such that almost no coordination with the DME system is needed.

The contribution of this paper is that it represents the first work fulfilling the requirements of [3] [4] regarding the spectrum sharing in the frequency range $960-1164 \mathrm{MHz}$ and achieving full coverage of LDACS in Europe without imposing any changes on the DME system. Neither signaling between primary and secondary users, nor a centralized controller as in [22] is required. Additionally, worst-case scenarios have been assumed. Following a DME-compliant LDACS cell planning as the one presented in this paper, LDACS transmitters could access, anywhere in Europe and at any time, parts of the licensed spectrum (960-1164 MHz) without disturbing the proper operation of the primary DME system.

The remainder of this paper is organized as follows: In Section II we shortly introduce the DME system. This is followed by necessary mathematical preliminaries in Section III. In Section IV, the performance of DME devices under LDACS interference is shown. In Section $\mathrm{V}$ and $\mathrm{VI}$ we introduce spectral, spatial and power separation between LDACS-FL and DME signals which guarantee a proper operation of DME devices under the existence of LDACS interference. A result set of DME-compliant locations for LDACS ground-stations and channel assignments is given in Section VII Simulation results are shown in Section VIII. We finish this paper with a conclusion in Section IX

\section{Distance Measuring Equipment}

The DME system aims to measure the slant range between aircraft and ground stations. The DME system consists of two classes of devices: an interrogator at the aircraft and a transponder at the ground station. The interrogator transmits Gaussian shaped pulse pairs (randomly spaced in time) with a certain rate. After detecting the interrogation signal at the transponder, a pair of Gaussian shaped pulses are transmitted after a known time offset on a frequency located at $\pm 63 \mathrm{MHz}$ from the frequency of the interrogator signal depending on the DME mode (X or Y). By knowing the propagation delay between the airborne device and the ground station (and the time offset), the slant range can be directly estimated. DME channels for both $\mathrm{X}$ and $\mathrm{Y}$ modes are given in Tab. I

TABLE I

DME CHANNELS FOR BOTH X AND Y MODE.

\begin{tabular}{|c|c|c|}
\hline DME Channel & $\begin{array}{c}\text { Interrogation Frequency } \\
\mathrm{MHz}\end{array}$ & $\begin{array}{c}\text { Transponder Frequency } \\
\mathrm{MHz}\end{array}$ \\
\hline $1 \mathrm{X}-63 \mathrm{X}$ & $1025-1087$ & $962-1024$ \\
\hline $64 \mathrm{X}-126 \mathrm{X}$ & $1088-1150$ & $1151-1213$ \\
\hline $1 \mathrm{Y}-63 \mathrm{Y}$ & $1025-1087$ & $1088-1150$ \\
\hline $64 \mathrm{Y}-126 \mathrm{Y}$ & $1088-1150$ & $1025-1087$ \\
\hline
\end{tabular}

We notice from Tab. I that the sharing conditions in the frequency band $1025-1150 \mathrm{MHz}$ are more complex since both 
transponders and interrogators act as receivers. This has been already recognized in [3].

\section{Designated Operation Conditions (DOC) for DME Transpon- ders}

Each DME transponder with a given equivalent isotropically radiated power $E I R P_{T}[\mathrm{dBW}]$ has its own:

- maximum designated operational range (radius) $R_{D M E}$ expressed usually in nautical miles (NMi).

- maximum designated operational height $h_{D M E}$ expressed in hundreds of feet above the sea level. $h_{D M E} \ll R_{D M E}$.

Remark 1. The tactical air navigation (TACAN) system, the military variant of DME, is also taken into account in our analysis. The term DME is used in this paper for both DME and TACAN systems.

\section{Mathematical PRELIMINARIES}

In this section we introduce mathematical definitions and relations that will be used throughout this paper. Proofs and supporting lemmas can be found in Appendix C.

\section{A. Definitions}

- A candidate location for an LDACS GS is a geographical location intended to act as a location for an LDACS GS.

- A possible location for an LDACS GS is a candidate location associated with at least one FL channel such that the proper operation of the DME system is not disturbed.

- An allocated location for an LDACS GS is a possible location which additionally fulfills predefined interference constraints within LDACS itself.

- In this paper, unless mentioned explicitly, distances are in $\mathrm{km}$, frequencies (and frequency shifts) are in $\mathrm{MHz}$.

\section{B. Radio Visibility for Line-of-Sight Propagation}

All ground stations within a circle of radius $R_{h}$, ignoring the terrain, are radio visible by an aircraft $c$ flying at an altitude $h$, cf. Fig. 11. The relation between $h$ and $R_{h}$ can be given as [23]

$$
R_{h}=130.4 \cdot\left(\sqrt{h}+\sqrt{h_{g}}\right) .
$$

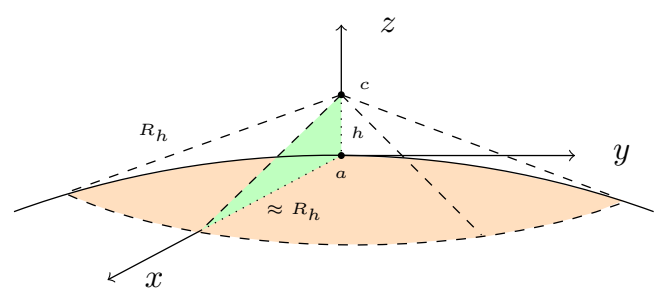

Fig. 1. Radio visibility of a flying aircraft $c$ at an altitude $h$ (exaggerated)

In the last relation it has been assumed that

- The radius of the earth $R_{e}=6378.137$.

- The radius of the earth is scaled by a factor of $\frac{4}{3}$ to count for refraction in the atmosphere.

- $h_{g}$ is the altitude of the ground station.
Because $R_{e} \gg R_{h}$ for realistic $h$ and $h_{g}$ we assume for the rest of this paper that all points on the spherical cap of radius $d_{\max }$ are located on a plane. $d_{\max }$ is not shown in Fig. 1, but explained in detail below. This reduces the spherical cap in Fig. 1 to a circle. In this paper, geodetic coordinate system data (as used by GPS) are converted to earth-centered earth-fixed coordinate system (ECEF) based on WGS84 ellipse model [24].

\section{Basic Distance Quantities}

Fig. 2 shows the service range which is the inner cylinder with operation range $R_{D M E}$ and operation height $h_{D M E}$ of a DME transponder located at vertex $b$. For every DME transponder we define:

$d_{\text {min }}=2 \cdot 130.4 \cdot \sqrt{h_{g}}$,
$d_{\text {max }}=R_{D M E}+\sqrt{130.4^{2} \cdot\left(\sqrt{h_{D M E}}+\sqrt{h_{g}}\right)^{2}-h_{D M E}^{2}}$.

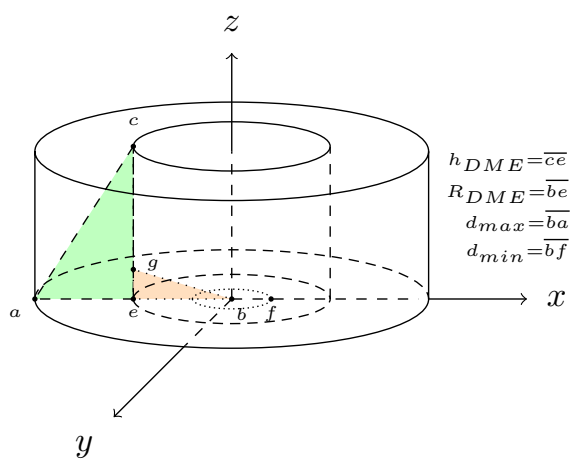

Fig. 2. Service range of a DME transponder and other important distances.

All LDACS GSs are "radio" visible by a DME transponder as long as the distances in-between are up to $d_{\min }$. Moreover, all LDACS GSs within a radius of $d_{\max }$ centered at the DME transponder $b$ are "radio" visible by an aircraft flying on the border of the service range of the DME transponder located at $b$ at the operation height $h_{D M E}$.

\section{Maximum Squared Distance Ratio in the Service Range of DME Transponders}

Given that $d$ is the distance between a DME transponder and an LDACS GS, we distinguish several cases according to the maximum squared distance ratio $\phi_{\max }$ (cf. Eq. 18 in Appendix C) between the DME transponder and the aircraft, and the LDACS ground station and the aircraft inside the service range of the DME transponder. $\phi$ is discussed in detail in Appendix C.

- $d>d_{\text {max }}$ : there is no radio visibility between the aircraft (within the DME transponder service range) and the LDACS GS. Thus, no coordination between the LDACS GS and this DME transponder is required.

- $2 \cdot R_{D M E} \leq d \leq d_{\max } \Rightarrow \frac{d}{2} \geq R_{D M E}$ : in this case $\phi_{\max }=$ 1 based on Rem. 14 This represents a condition in favor of the DME system since $\phi(x, y, z)$ in this case within the service range of the DME transponder is always smaller than 
1 and it equals 1 only for $d=2 \cdot R_{D M E}$ (if the aircraft is at the border of the service range of the DME transponder).

- $R_{D M E}<d<2 \cdot R_{D M E}$ : our simulations show that $\phi_{\max }$ in this case can be well approximated by $\frac{R_{D M E}^{2}+h_{D M E}^{2}}{\left(d-R_{D M E}\right)^{2}+h_{b r}^{2}}$. $h_{b r}$ represents the minimum $z \in\left[h_{\min }, h_{D M E}\right]$ such that

$$
130.4^{2} \cdot\left(\sqrt{z}+\sqrt{h_{g}}\right)^{2} \geq z^{2}+\left(d-R_{D M E}\right)^{2},
$$

where $h_{\min }$ is the minimal aircraft flying altitude.

- $0<d \leq R_{D M E}$ : in this case we define $\breve{h}_{\text {min }}$ as the smallest $z \in\left[h_{m i n}, h_{D M E}\right]$ which fulfills

$$
130.4^{2} \cdot\left(\sqrt{z}+\sqrt{h_{g}}\right)^{2} \geq z^{2}+\breve{x}_{2}^{2} .
$$

$\phi_{\max }$ in this case is given in Eq. 29b, (31) for $h_{\min }=$ $\breve{h}_{\min }$ which will be defined numerically within a resolution of 1 meter. $\breve{x}_{2}$ can be obtained using Eq. 30c).

Remark 2. In the following we assume:

- All LDACS GSs and all DME transponders are located at the same height $h_{g}=0.01$.

- $h_{\min }=3.048 \gg h_{g}$. This corresponds to FL100 1

- $h_{\max }=18.288$. This corresponds to FL600.

- Power of DME signals is peak power, whereas the power of LDACS signals is average power.

\section{Performance of DME Devices Under LDACS INTERFERENCE}

The performance of DME devices in the presence of LDACS signals has been investigated in [18]. Minimum required $\frac{D}{U}[\mathrm{~dB}]$ for proper operation of DME devices as a function of the spectral separation between the LDACS transmit channel and the receive channel of the DME device has been identified. $D$ (abbreviated from desired) represents the receive power at the input of the interrogator/transponder, where the transmitter is the transponder/interrogator. $U$ (abbreviated from undesired) represents the LDACS signal power received at the input of the interrogator/transponder. Since we are interested in the cell planning for LDACS FL, we focus on the case, where the LDACS GS generates the interference signal towards DME devices.

Unfortunately, no measurements have been performed for spectral separation higher than $3 \mathrm{MHz}$ for DME interrogators and $3.5 \mathrm{MHz}$ for DME transponders, respectively, except for $93 \mathrm{MHz}$. For $93 \mathrm{MHz}$ spectral separation, no influence of the LDACS FL signal upon DME devices has been registered. For the lack of measurements we define in this paper $\Delta f_{I, S t o p}$ and $\Delta f_{T, S t o p}$ as in Tab. II for DME interrogator and Tab. III for DME transponders, respectively.

TABLE II

MINIMUM REQUIRED $\frac{D}{U}$ FOR DME INTERROGATORS AS A FUNCTION OF THE SPECTRAL SEPARATION.

\begin{tabular}{|c|c|c|c|c|c|c|c|}
\hline$\left|\Delta f_{\text {offset }}\right|[\mathrm{MHz}]$ & 0 & 0.5 & 1 & 1.5 & 2 & 2.5 & $\begin{array}{c}\geq 3 \\
\end{array}$ \\
\hline$\beta_{I}=\frac{D}{U}[\mathrm{~dB}]$ & 17 & 2 & -31 & -49 & -49 & -55 & -60 \\
\hline
\end{tabular}

${ }^{1} 1$ Flight Level (FL) is equivalent to $100 \mathrm{ft}$ of altitude e.g. FL100 corresponds to $10,000 \mathrm{ft}$ altitude.
TABLE III

MINIMUM REQUIRED $\frac{D}{U}$ FOR DME TRANSPONDERS AS A FUNCTION OF THE SPECTRAL SEPARATION.

\begin{tabular}{|c|c|c|c|c|c|c|c|c|}
\hline$\left|\Delta f_{\text {offset }}\right|[\mathrm{MHz}]$ & 0 & 0.5 & 1 & 1.5 & 2 & 2.5 & 3 & $\begin{array}{c}\geq 3.5 \\
<f_{T, \text { Stop }}\end{array}$ \\
\hline$\beta_{T}=\frac{D}{U}[\mathrm{~dB}]$ & 38 & 31 & 23 & 14 & 5 & -4 & -14 & -15 \\
\hline
\end{tabular}

Particularly, the case $\Delta f_{I, \text { Stop }}=\Delta f_{T, \text { Stop }}=93 \mathrm{MHz}$ in the last column of Tab. II. III represents a very conservative condition in favor of the DME system (worst case for LDACS). It says that, the minimum required $\frac{D}{U}$ does not decrease any more by increasing the frequency offset between LDACS FL signal and DME receive channel beyond 3 (3.5) $\mathrm{MHz}$ up to $93 \mathrm{MHz}$. On the other hand, the best case for LDACS is for $\Delta f_{I, \text { Stop }}=3.5 \mathrm{MHz}$ and $\Delta f_{T, \text { Stop }}=4 \mathrm{MHz}$. Tab. III includes the TACAN system as well.

Remark 3. The criteria which define the proper operation of DME devices are given in [18].

\section{Converting Minimum Required $\beta_{I}=\frac{D}{U}$ TO SPATIAL AND SPECTRAL SEPARATION FOR DME INTERROGATORS}

From Fig. 11 we notice at $c$ assuming a free space line-ofsight propagation:

$$
\begin{aligned}
D[\mathrm{dBm}] & =E I R P_{T}[\mathrm{dBW}]+30+G_{I}[\mathrm{dBi}] \\
& -20 \cdot \log \left(d_{2}\right)-20 \cdot \log \left(f_{T}\right)-32.45 . \\
U[\mathrm{dBm}] & =E I R P_{L}[\mathrm{dBm}]+G_{I}[\mathrm{dBi}] \\
& -20 \cdot \log \left(d_{1}\right)-20 \cdot \log \left(f_{L}\right)-32.45 .
\end{aligned}
$$

In Eq. (5), (6)

- $E I R P_{T}$ : Equivalent isotropically radiated power of the $\mathrm{DME}$ transponder, usually given in $\mathrm{dBW}$. It includes the transmit antenna gain and the cable losses.

- EIRP $P_{L}$ : Equivalent isotropically radiated power of the LDACS GS in $\mathrm{dBm}$. It includes the transmit antenna gain and the cable losses. For the en-route case, $\operatorname{EIRP}_{L} \in$ $\{42,47,52\}$ corresponding to cell size $\in\{60,120,200\}$ NMi, respectively [8].

- $G_{I}$ : The gain of the receive antenna of the interrogator in $\mathrm{dBi}$.

- $d_{2}$ : The distance from transponder to interrogator.

- $d_{1}$ : The distance from LDACS GS to interrogator.

- $f_{T}$ : Transmit frequency of the transponder (receive frequency of the interrogator).

- $f_{L}$ : Transmit frequency of the LDACS GS.

Based on Eq. (5), (6) we find that

$$
\begin{aligned}
\frac{D}{U}[\mathrm{~dB}] & =D[\mathrm{dBm}]-U[\mathrm{dBm}] \\
& =30+E I R P_{T}[\mathrm{dBW}]-E I R P_{L}[\mathrm{dBm}] \\
& -20 \cdot \log \frac{d_{2}}{d_{1}}-20 \cdot \log \frac{f_{T}}{f_{L}}
\end{aligned}
$$

However, depending on Tab. II

$$
\frac{D}{U}[\mathrm{~dB}] \geq \beta_{I}\left(\Delta f_{\text {offset }}=\left|f_{T}-f_{L}\right|\right)
$$


This leads depending on Sec. III-D to

$$
\begin{aligned}
E I R P_{L}[\mathrm{dBm}]+\beta_{I}\left(\Delta f_{\text {offset }}\right) \leq & E I R P_{T}[\mathrm{dBW}]+30 \\
& -10 \cdot \log \left(\phi_{\max }\right)-|\zeta| \\
& -20 \cdot \log \frac{f_{T}}{f_{L}} .
\end{aligned}
$$

Eq. (9) indicates that in order to establish an LDACS GS without influencing the proper operation of DME interrogators, we need to equip the LDACS GS with $E I R P_{L}$ and $f_{L}$ such that Eq. (9) is fulfilled for all DME transponders which are as far as $d_{\max }$ in Eq. (2b) from the LDACS GS.

Remark 4. During deriving Eq. (9) we have assumed that the interrogator antenna gain is the same for both LDACS GS and DME transponder signals. However, this is true only in the co-site case (LDACS GS and DME transponder at the same location). For other cases, the interrogator antenna pattern must be taken into account. To compensate this effect, we introduce in this paper the constant factor $|\zeta|$ as a margin in favor of the DME system.

\section{Co-Site Case:}

In a special case where the LDACS GS is located at the same location as the DME transponder Eq. 97) (for this DME transponder) can be relaxed to, cf. Rem. 13

$$
\begin{aligned}
E I R P_{L}[\mathrm{dBm}]+\beta\left(\Delta f_{\text {offset }}\right) & \leq E I R P_{T}[\mathrm{dBW}]+30-|\zeta| \\
& -20 \cdot \log \frac{f_{T}}{f_{L}} .
\end{aligned}
$$

\section{Vi. Converting Minimum Required $\beta_{T}=\frac{D}{U}$ to SPATIAL AND SPECTRAL SEPARATION FOR DME TRANSPONDERS}

In this case we assume $D[\mathrm{dBm}]=-96$. Further information related to this assumption are considered in Rem. 6

$$
\begin{aligned}
U[\mathrm{dBm}] & =E I R P_{L}[\mathrm{dBm}]+G_{T}[\mathrm{dBi}] \\
& -20 \cdot \log \left(d_{G}\right)-20 \cdot \log \left(f_{L}\right)-32.45 .
\end{aligned}
$$

$d_{G} \leq d_{\min }$ is the distance between the LDACS GS and the DME transponder located as far as $d_{\text {min }}$, cf. Eq. 2a. Following a similar approach as in Sec. ?? we obtain

$$
\begin{aligned}
E I R P_{L}[\mathrm{dBm}]+\beta_{T}\left(\Delta f_{\text {offset }}\right) \leq & -72.65-|\zeta| \\
& +20 \cdot \log \left(d_{G}\right)+20 \cdot \log \left(f_{L}\right) .
\end{aligned}
$$

Eq. (12) indicates that in order to establish an LDACS GS without influencing the proper operation of DME transponders, we need to equip the LDACS GS with $E I R P_{L}$ and $f_{L}$ such that Eq. (12) is fulfilled for all DME transponders which are as far as $d_{\min }$ in Eq. 2a) from the LDACS GS.

Remark 5. By deriving Eq. (12) we have assumed that the DME transponder's antenna gain regarding the LDACS FL signal is at maximum $G_{T}=9.1[\mathrm{dBi}][25]$. This is a strict assumption in favor of the DME system, since it is unlikely that the LDACS GS lies exactly in the maximum gain axis of the DME transponder's antenna.
Remark 6. The minimum required $\frac{D}{U}$ values in $[18]$ have been obtained for a $D$ slightly higher than $-96[\mathrm{dBm}]$. We assume that these values do not experience fundamental change for $D=-96[\mathrm{dBm}]$. We choose in this paper this value for $D$ since it is the minimum value at which long range DME transponders must still be capable to reply to an interrogation. Short range DME transponders require $D=-86[\mathrm{dBm}]$. More information can be found in [26, Sec. 3.5.4.2.3]. However, we assume in this paper $D=-96[\mathrm{dBm}]$ for all DME transponders regardless the short or long range operation. This represents also a conservative condition in the favor of the DME system.

\section{We conclude:}

For operating an LDACS GS without influencing the proper operation of the DME system, both Eq. 99, (12) must be fulfilled.

\section{LDACS POSSIBLE LOCATIONS AND CHANNEL ASSIGNMENTS}

This aims to define a set of possible locations for LDACS GSs out of a set of candidate locations with associated channel assignment and cell size such that

1) The proper operation of the DME system is not affected, i.e. Eq. (99, (12) are fulfilled.

2) The co-channel interference within LDACS itself is limited.

3) Complete coverage for the desired area is achieved.

For "common" frequency-reuse systems such as the mobile phone system, where the frequency band is completely reserved for that service, the first item is not required.

\section{A. Step 1: LDACS GS Possible Locations}

In this step, two infrastructure lists are provided. The first one contains the DME data, such as location, channel, DOC and EIRP. The second infrastructure list is the frequency list and contains the candidate locations. A list of LDACS FL channels is also given. Locations are given in GPS-format and are converted into ECEF-format [24]. The algorithm in Step 1 delivers the binary matrices $\boldsymbol{Z}_{1}, \boldsymbol{Z}_{2}, \cdots, \boldsymbol{Z}_{S}$ of size $L \times C$. $S$ is the number of possible LDACS cell sizes. $L$ represents the number of candidate locations and $C$ is the number of provided LDACS FL channels.

The algorithm in Step 1 proceeds for given $h_{\min }, h_{\max }$ as follows:

- Initialize $\boldsymbol{Z}_{1}=\boldsymbol{Z}_{2}=\cdots=\boldsymbol{Z}_{S}=\mathbf{0}_{L \times C}$.

- Calculate $d_{\min }$ Eq. (2a).

- Calculate $d_{\max }$ Eq. (2b) for each DME transponder.

- For each candidate location $i \in\{1,2, \cdots, L\}$, each LDACS FL channel $j \in\{1,2, \cdots, C\}$ and each cell size $s \in$ $\{1,2, \cdots, S\}$

1) Calculate the distance $d$ between the candidate location $i$ and all DME transponders. The case $d=0$ is replaced by $d=0.025$.

2) Define Set S1 which contains all DME transponders where $d \leq d_{\min }$. 
3) Define Set S2 which contains all DME transponders where $d \leq d_{\max }$.

4) For the DME transponders in Set $\mathrm{S} 2$ calculate $\phi_{\max }$ based on Sec. III-D

5) If Eq. (12) is fulfilled for all DME transponders in Set S1 and Eq. (9) is fulfilled for all DME transponders in Set $\mathbf{S} 2$, then $\boldsymbol{Z}_{s}(i, j)=1$.

The DME data is available in the so-called COM3-Table. This table is periodically published by the International Civil Aviation Organization (ICAO), cf. Appendix A We assume that the LDACS GS candidate locations are the current locations of the aeronautical VHF-stations. The data of these locations are periodically published by ICAO as COM2-Table, cf. Appendix B The total number of DME transponders is 2371 and the number of candidate locations is 3248 . However, they are not uniformly distributed.

The results are shown in Fig. 3, 4 for $\Delta f_{I, \text { Stop }}=$ $\Delta f_{T, S t o p}=93 \mathrm{MHz}$ (worst case) and $\Delta f_{I, S t o p}=3.5 \mathrm{MHz}$, $\Delta f_{T, S t o p}=4 \mathrm{MHz}$ (best case), respectively. The vertical axis in Fig. 3, 4 represents the percentage of the 3248 candidate locations where the proper operation of the DME system is not affected as a function of LDACS FL transmit frequency and cell size (in NMi). We distinguish between onlay deployment $f_{L} \in\{960: 1: 1164\} \mathrm{MHz}$, cf. Fig. 3(a), 4(a) and inlay deployment $f_{L} \in\{960.5: 1: 1163.5\}$ MHz, cf. Fig. 3(b) 4(b) In all cases $\zeta=10 \mathrm{~dB}$.

Remark 7. $\zeta$ counts also for the combined influence of many LDACS GSs on the same DME transponder/interrogator, cf. Rem 4

Based on Fig. 3, 4 we conclude:

- Decreasing $\Delta f_{I, \text { Stop }}$ and/or $\Delta f_{T, S t o p}$ increases the number of possible locations.

- For the onlay deployment, the influence of the cell size is negligible because of the utilization of $\beta_{I}(\Delta f=0)$ and $\beta_{T}(\Delta f=0)$ as in Tab. II III] since DME channels are located on a $1 \mathrm{MHz}$ grid in the frequency range 962-1213 MHz.

- For frequency bands with poor/no DME allocations there is a negligible difference between the inlay and onlay deployment. These are the frequency bands [960 - 978] $\mathrm{MHz}$, around $1030 \mathrm{MHz}$, around $1090 \mathrm{MHz}$ and around $1153 \mathrm{MHz}$ (i.e. $1090 \mathrm{MHz}+63 \mathrm{MHz}$ ).

- For frequency bands with poor/no DME allocations the influence of the cell size is negligible.

- For frequency bands with rich DME allocations, the number of possible locations increases by decreasing the cell size since this decreases the interference power towards DME devices.

- Although not shown here, decreasing $|\zeta|$ increases the number of possible locations and vice versa.

Remark 8. Because LDACS utilizes frequency-division duplexing, we assume in the following that the LDACS RL is deployed around $965 \mathrm{MHz}$ which is effectively free of DME allocations and does not require an extensive coordination. For the LDACS RL, a combination of inlay and onlay deployment can be applied. This increases the number of available chan-

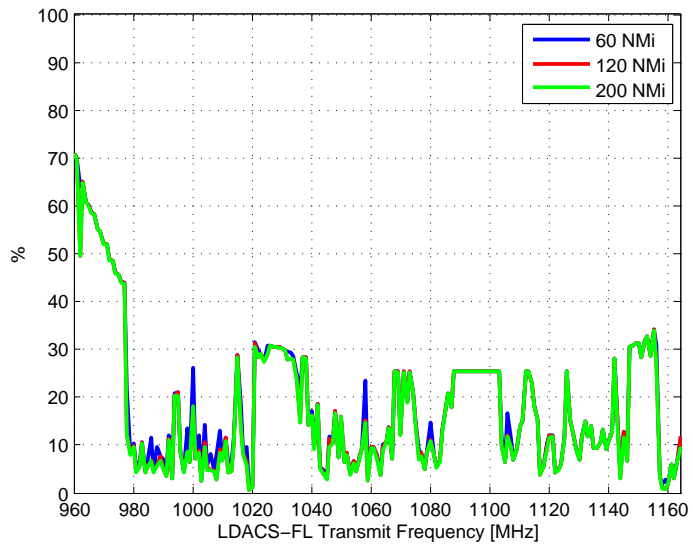

(a) Onlay case $f_{L} \in\{960: 1: 1164\} \mathrm{MHz}$.

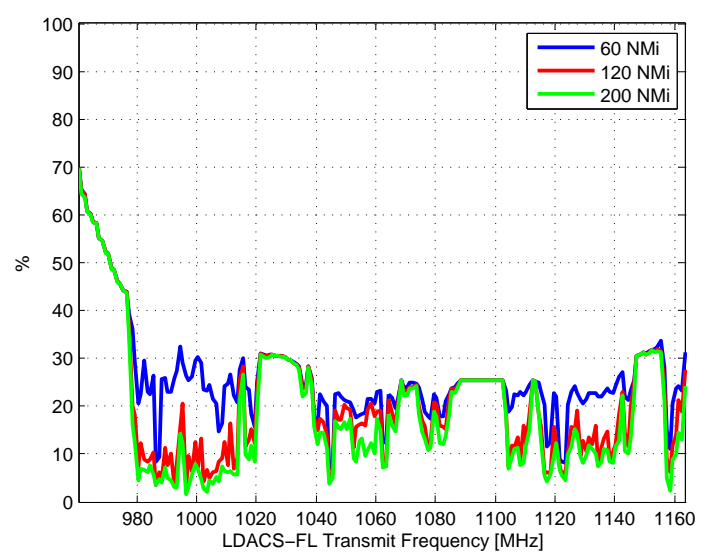

(b) Inlay case $f_{L} \in\{960.5: 1: 1163.5\} \mathrm{MHz}$.

Fig. 3. The percentage of the 3248 candidate locations where the proper operation of the DME system is not affected as a function of LDACS FL channel and cell size (in NMi). $\Delta f_{I, S t o p}=\Delta f_{T, S t o p}=93 \mathrm{MHz} . \zeta=10$ dB.

nels for LDACS RL. For LDACS FL we focus on the inlay case. Based on Fig. 3(b) we notice that the sub-band around $1055 \mathrm{MHz}$ is promising for the LDACS FL. In the following, channel assignment for LDACS FL is performed in the subband 1048.5: $1: 1060.5 \mathrm{MHz}$. By this assignment the spectral separation of the LDACS FL and LDACS RL channels from the secondary surveillance radar (SSR) channels at 1030 and $1090 \mathrm{MHz}$ are taken into account.

\section{B. Step 2: LDACS FL Channel Assignments}

Co-channel interference in frequency reuse systems decreases the overall system performance [27] and must be minimized as an example by a proper geographical separation between cells with the same channel assignment. We assume in the following that all cells have the same size $s$ and we define $\boldsymbol{R}=\boldsymbol{Z}_{s}$, which is the output of Step $\boldsymbol{l}$ for the cell size $s$. In the rest of this paper $l, i \in\{1,2, \cdots, L\}$ and $c, j \in\{1,2, \cdots, C\}$. We define the following interference constraints:

- At a possible location $i$, different channel assignments $j \neq c$ are simultaneously allowed as long as $|j-c| \geq \eta_{1}$. In this case $\eta_{1} \mathrm{MHz}$ represents the smallest frequency offset that must be fulfilled such that operating both channels $j$ and $c$ at the same possible location $i$ is allowed. This presumes a 


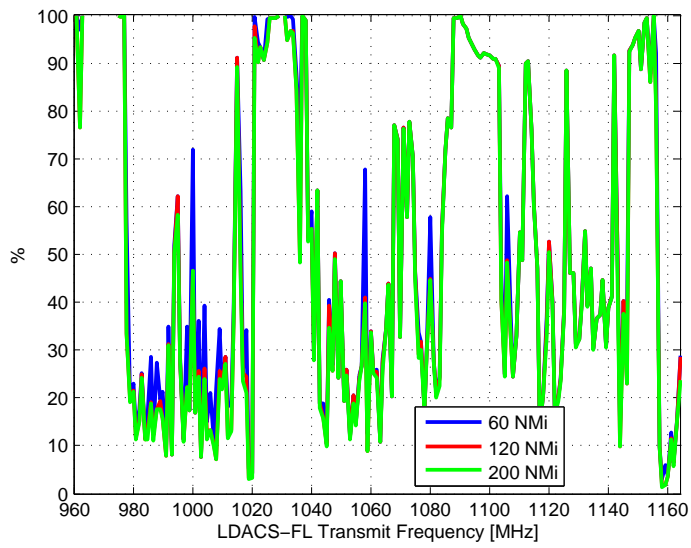

(a) Onlay case $f_{L} \in\{960: 1: 1164\} \mathrm{MHz}$.

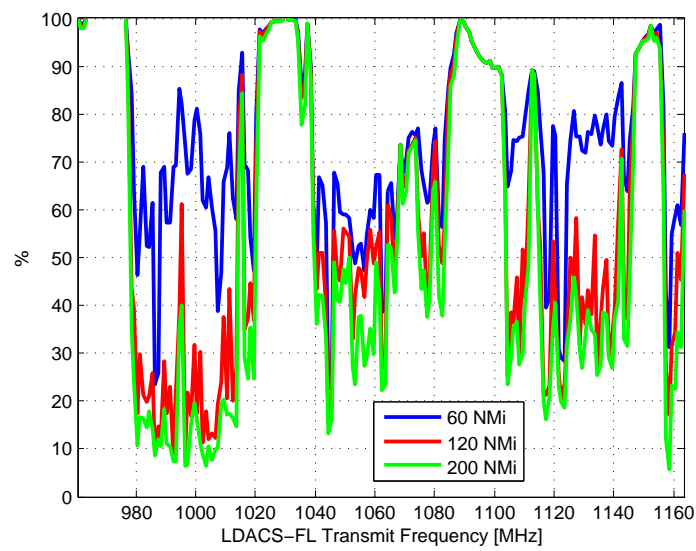

(b) Inlay case $f_{L} \in\{960.5: 1: 1163.5\} \mathrm{MHz}$.

Fig. 4. The percentage of the 3248 candidate locations where the proper operation of the DME system is not affected as a function of LDACS FL channel and cell size (in NMi). $\Delta f_{I, S t o p}=3.5 \mathrm{MHz}$ and $\Delta f_{T, S t o p}=4$ MHz. $\zeta=10 \mathrm{~dB}$

contiguous spectrum. However, this condition can be easily modified for a non-contiguous spectrum. $\eta_{1}$ is integer and $\eta_{1} \in\{1,2, \cdots, C\}$. For $\eta_{1}=C$, up to one channel is permitted to operate at each possible location.

- At different possible locations $l \neq i$, the same channel $j$ is allowed to operate as long as the distance $d_{l i}$ between location $l$ and location $i$ is greater or equal to a reference distance $d_{c o}$ (co-channel distance).

- At different possible locations $l \neq i$, different channel assignments $j \neq c$ are allowed to operate as long as the distance $d_{l i}$ fulfills the following relation

$$
d_{l i} \geq\left\{\begin{array}{cl}
0 & :|j-c| \geq \eta_{2} \\
d_{c o} \cdot\left(1-\frac{|j-c|}{\eta_{2}}\right) & :|j-c|<\eta_{2}
\end{array}\right.
$$

The aim of Step 2 is to deliver $\hat{\boldsymbol{R}}$ : LDACS GS locations with certain frequency assignments such that the above mentioned interference constraints are never violated. For this purpose, we utilize a graph based approach.

1) Interference Graph: Graph based approaches for channel assignment have been already utilized, cf. [28] and the references therein. Our contribution is to put the graph based approach in the context of LDACS FL and to connect the interference constraints with the resulting graph. The channel assignment for LDACS FL is equivalent to finding the maximum independent set in a graph. This is solved by applying a two-state Hopfield network. Modifications to an already known solution [21], especially stability and priority aspects are presented, too.

Consider a graph $\hat{\mathcal{G}}(\hat{\mathcal{V}}, \hat{\mathcal{E}})$ consisting of $L \cdot C$ vertices. Each vertex represents a candidate location $l$ with a channel assignment $c$. Vertices with $\boldsymbol{R}(l, c)=0$ are excluded from the graph $\hat{\mathcal{G}}$ since they disturb the proper operation of the DME system. The resulting graph $\mathcal{G}(\mathcal{V}, \mathcal{E})$ includes only possible locations associated with DME-compliant transmit channels. Vertex $\tilde{l}$ is connected to vertex $\tilde{i}$ if operating an LDACS GS at $\tilde{l}$ and $\tilde{i}$ simultaneously violates at least one of the above mentioned interference constraints. In this case, finding a set of LDACS GS locations with suitable frequency assignments which do not violate the interference constraints is nothing else than finding an independent set in the graph $\mathcal{G}$ i.e. finding a subset of vertices which do not share any edges. This is an NP-complete problem [20]. We apply Hopfield networks as dynamical solvers (without any need for training) for providing an independent set of the interference graph $\mathcal{G}$.

2) Two-State Hopfield Network: Fig. 5 shows exemplary a two-state Hopfield network [29] arranged in a grid. It consists of ordered nodes (so called neurons) and weighted connections in-between. The computational concept of the neuron in the $i$ th row and $j$-th column is described by the following equations

$$
\begin{aligned}
& u_{i j}[\kappa]=\sum_{l=1}^{L} \sum_{c=1}^{C} w_{l c, i j} \cdot v_{l c}[\kappa-1], \\
& v_{i j}[\kappa]=\theta\left(u_{i j}[\kappa]\right), \\
& \theta\left(u_{i j}\right)=\left\{\begin{array}{lll}
1 & \text { for } u_{i j} \geqq 0, \\
0 & \text { for } u_{i j}<0 .
\end{array}\right.
\end{aligned}
$$

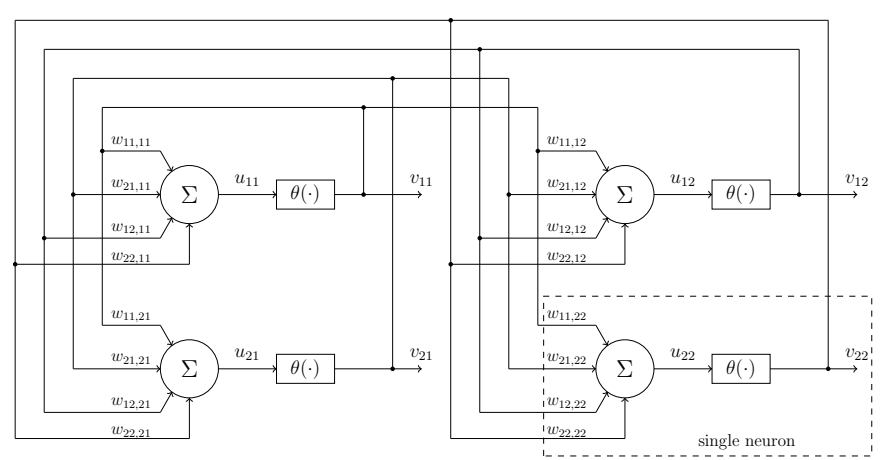

Fig. 5. Hopfield Network arranged in a grid, exemplary four neurons.

In Eq. 14 and Fig. 5, $u_{i j}$ and $v_{i j}$ are the inner state and the output of the neuron in the $i$-th row and $j$-th column, respectively. $L / C$ is the number of neurons in one column/row. $w_{l c, i j}$ represents the weight coefficient from the output of the neuron in the $l$-th row and $c$-th column to the input of the neuron in the $i$-th row and $j$-th column. $\kappa$ is the discrete-time iteration variable and $\theta$ represents the activation function (in this case the unit step function). We assume an asynchronous update mode (at each $\kappa$ only one neuron is 
updated). This is because of stability aspects and is discussed later in Sec. VII-B4 The influence of the update order and its relation to priority aspects is discussed in Sec. VIII-A

The Hopfield network has been applied to solve many combinatorial optimization problems. One of the first and most well known applications of Hopfield network is the content addressable memory [29], [30]. Other applications are A/D converter [31], the traveling salesman problem [32] and channel equalization [33], among others. In all these applications no training is needed. The Hopfield network is utilized as a "dynamical solver". We adapt this approach to find an independent set of the graph $\mathcal{G}$ resulting from Sec. VII-B1.

3) LDACS Channel Assignment based on the Two-State Hopfield Network: For this purpose we need to define

- The weight coefficients.

- The initial states, i.e. outputs and inner states at $\kappa=0$.

- Update order because of the asynchronous update mode.

- Stopping criterion.

We notice that the number of neurons in a column equals $L$, the number of candidate locations, whereas the number of neurons in a row equals $C$, the number of available LDACS FL channels. We define the weights $w_{l c, i j}$ as follows:

Case 1: Horizontal Connections $l=i$ : This defines the connections between the neurons representing the same candidate location and is the same for all candidate locations

$$
w_{i c, i j}=\left\{\begin{aligned}
0 & :|j-c| \geq \eta_{1} \quad \text { or } j=c \\
-1 & : \text { Otherwise }
\end{aligned}\right.
$$

In a special case where $\eta_{1}=C$ we obtain:

$$
w_{i c, i j}=\left\{\begin{aligned}
0 & : j=c \\
-1 & : \text { Otherwise. }
\end{aligned}\right.
$$

Case 2: Cross and Vertical Connections $l \neq i$ : This defines the connections between the neurons representing different candidate locations.

$w_{l c, i j}=\left\{\begin{aligned} 0 & :|j-c| \geq \eta_{2}, \\ & \text { or } d_{l i}>d_{h}^{(L)}, \\ & \text { or }|j-c|<\eta_{2} \text { and } d_{l i}-d_{c o} \cdot\left(1-\frac{|j-c|}{\eta_{2}}\right) \geq 0 \\ -1 \quad: \text { Otherwise. } & \end{aligned}\right.$

$w_{l c, i j}=0$ means that there is no need for coordination between the candidate locations $l$ and $i$ if the $c$-th channel is assigned to the $l$-th location and $j$-th channel is assigned to the $i$-th location.

$w_{l c, i j}=-1$ means that assigning the $c$-th channel to the $l$ th location, and at the same time the $j$-th channel to the $i$ th location is not possible since it violates the interference constraints. The weight coefficients $w_{l c, i j}$ are comparable to the edges of the interference graph $\hat{\mathcal{G}}$.

$d_{h}^{(L)}$ is the minimum distance between the candidate locations $l, i$ such that the LDACS GS at location $l$ is not "radio" visible by an aircraft flying at the maximum possible flight level on the border of the LDACS GS cell centered at location $i$

$$
d_{h}^{(L)}=R_{L D A C S}+\sqrt{130.4^{2} \cdot\left(\sqrt{h_{\max }}+\sqrt{h_{g}}\right)^{2}-h_{\max }^{2}} .
$$

$R_{L D A C S}$ is the radius of the LDACS cell. In addition $d_{c o} \leq$ $d_{h}^{(L)}$. Moreover, we define the initial states as

$$
\begin{aligned}
& \boldsymbol{V}[\kappa=0]=\left\{v_{l c}: \forall l \in\{1,2, \cdots, L\}, \forall c \in\right. \\
& \{1,2, \cdots, C\}=\boldsymbol{R} . \\
& \text { - } \boldsymbol{U}[\kappa=0]=\left\{u_{l c}: \forall l \in\{1,2, \cdots, L\}, \forall c \in\right. \\
& \{1,2, \cdots, C\}=\mathbf{0} .
\end{aligned}
$$

Furthermore, a neuron in the $l$-th row and $c$-th column where $v_{l c}[\kappa=0]=0$, i.e. $\boldsymbol{R}(l, c)=0$ will never be updated. This is comparable to the movement from $\hat{\mathcal{G}}$ to $\mathcal{G}$ (thus considering only possible locations and not candidate ones). All other neurons are updated randomly but with equal probability.

Remark 9. Based on Eq. (15), (16) we notice that

- $w_{l c, i j}=w_{i j, l c}$ : symmetric weight coefficients.

- $w_{i j, i j}=0$, no self feedback.

4) Lyapunov Stability: The asynchronous updated Hopfield network in Eq. (14) is locally asymptotically stable in the sense of Lyapunov [30] if the weight coefficients are symmetric and the self feedback is non-negative. Both conditions are fulfilled in our case, cf. Rem 9. This has been proved in [29], [33], [34]. The needed number of iterations (upper bound) to reach a fixed point has also been given in [33] and depends primarily on the minimum and maximum eigenvalues of the weight matrix. We avoid the synchronous update, where all neurons are updated at the same time because of the limit cycle issue: Synchronous updated Hopfield networks can reach a limit cycle of length two [33], [35].

By evaluating Eq. (14) taking into account Eq. (15), 16 $\boldsymbol{V}$ reaches a fixed point $\boldsymbol{V}_{f p}$ and any further update is useless. The output of Step 2 is $\hat{\boldsymbol{R}}=\boldsymbol{V}_{f p}$ and represents an independent set of the interference graph $\mathcal{G} . \hat{\boldsymbol{R}}$ is the result of the cell planning and contains

- The locations of LDACS GSs which do not disturb the proper operation of the DME system.

- The channel assignments of these locations such that the interference constraints are not violated.

\section{Simulation Results}

In this section we apply the method of Sec. VII-A and Sec. VII-B to perforam LDACS FL cell planning $\in\{1048.5$ : $1: 1060.5\} \mathrm{MHz}$. We split the update process of the Hopfield network into two phases

- Intra-location update: The neurons are updated in location order. For each location, vertical and cross weight coefficients are assumed to be zero. Neurons representing a possible location $i$ are updated randomly. The aim of this phase is to assign different channels $j \neq c$ to the same possible location $i$ such that $|j-c| \geq \eta_{1}$. This case is comparable with the situation where each location exists by one's lonesome. This step can be skipped for a possible location $i$ if $\sum_{c=1}^{C} \boldsymbol{R}(i, c) \leq 1$. 
- Extra-location update: The neurons are updated randomly with equal probability. Horizontal weight coefficients are assumed to be zero.

In both phases, iterations are performed till a fixed point is reached. Maximum required number of iterations for both phases can be calculated based on Theorem 10 in [33]. Separating the update process as suggested here is neither mandatory nor important for the asymptotical stability. However, the introduced update scheme is especially interesting for the case $\eta_{1}=1$. In this case, the intra-location update phase can be skipped. This is discussed in VIII-A

Remark 10. $\boldsymbol{R}(i, j)=0$ for a candidate location $i$ and channel assignment $j$, which is the output of Step 1, means that the $j$-th channel assignment at the $i$-th candidate location will disturb the proper operation of the DME system. Updating the neuron in the $i$-th row and $j$-th column of the Hopfield network in Step 2 could lead to a $\boldsymbol{R}(i, j)=1$, which is not allowed. Therefore, neurons in the $i$-th row and $j$-th column where $\boldsymbol{R}(i, j)=0$ do not experience the update process in Step 2 at all.

We conclude: The update process in Step 2 can flip a 1 to 0 (remove a channel assignment compared with the initial state $\boldsymbol{R}$ ) but not vice versa (establishing channel assignments compared with the initial state $\boldsymbol{R}$ is not allowed). In other words, Step 2 can change a possible location into a candidate one but not the way around.

In the following we show simulation results for cell size $R_{L D A C S}=120 \mathrm{NMi}$. LDACS FL transmit frequencies are $\{1048.5: 1: 1060.5\} \mathrm{MHz}$, so $C=13$. Moreover, $\zeta=10$ $\mathrm{dB}, \eta_{1}=C, d_{c o}=d_{h}^{(L)}$ and two values for $\eta_{2} \in\{1,2\}$. $\Delta f_{I, \text { Stop }}=\Delta f_{T, \text { Stop }}=93 \mathrm{MHz}$ (i.e. worse case). We notice that $d_{c o}=d_{h}^{(L)}$ is a conservative condition in favor of the DME system. In this case, the aircraft LDACS receiver has no radio visibility to other LDACS GS with the same channel as its own serving cell. For computational complexity purposes we randomly take a subset of the candidate locations: the minimum distance between any two possible locations is at least $35 \mathrm{Km}$. We repeat Step $210^{3}$ times (each time with different update order) and plot the average allocation of the LDACS FL channels $\in\{1048.5: 1: 1060.5\} \mathrm{MHz}$ in Fig. 6

The average number of allocated LDACS GSs is

- 83.939 for $\eta_{2}=1$

- 68.94 for $\eta_{2}=2$

An exemplary cell planning for LDACS FL is shown in Fig. 7(a) for $\eta_{2}=1$ and in Fig. 7(b) for $\eta_{2}=2$. In both cases, we notice the existence of enough assigned locations.

By repeating the simulations we observe

- Decreasing $\eta_{2}$ and/or $d_{c o}$ i.e. allowing more interference within LDACS increases the number of allocated LDACS GSs.

- The introduced method could allocate more LDACS GSs than needed for communications. This is not critical since removing cells is always possible.

- Small areas may stay not covered by an LDACS cell. This depends on the set of the candidate locations, which are in our case unequally distributed, cf. Appendix B

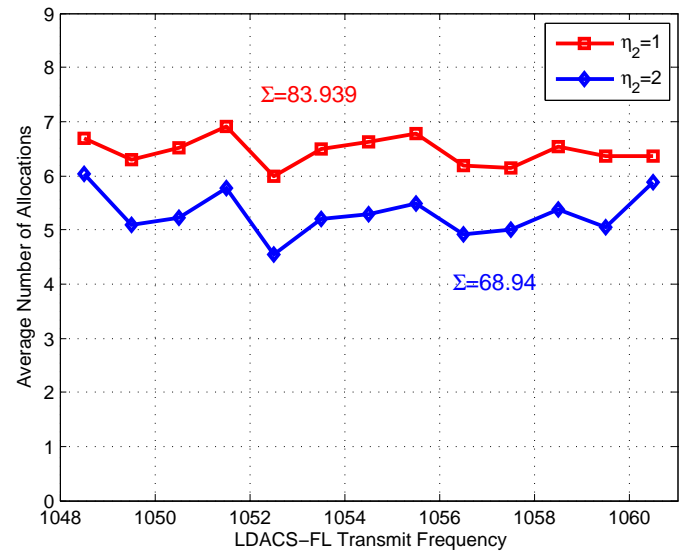

Fig. 6. Average (over $10^{3}$ runs) number of allocations for LDACS FL channels $\in\{1048.5: 1: 1060.5\} \mathrm{MHz}$ for $\eta_{1}=13, \eta_{2} \in\{1,2\}$. Cell size $R_{L D A C S}=120 \mathrm{NMi}, h_{g}=0.01, h_{\min }=0.3048, h_{\max }=18.288$ $\zeta=10, d_{c o}=d_{h}^{(L)} . \Delta f_{I, \text { Stop }}=\Delta f_{T, \text { Stop }}=93 \mathrm{MHz}$

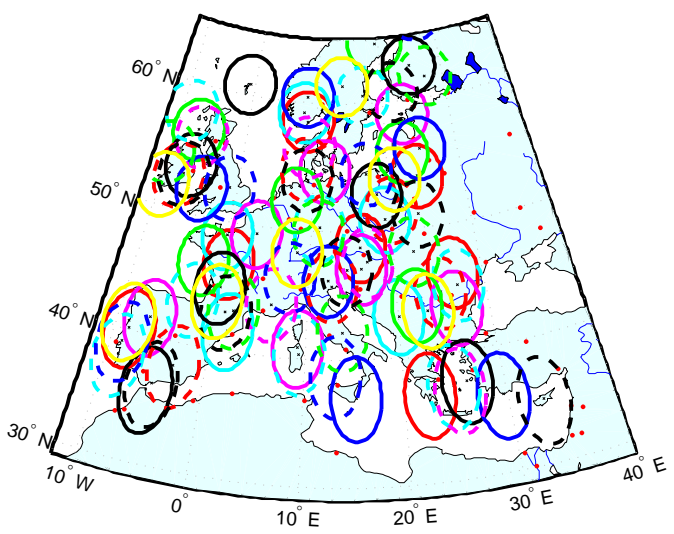

(a) $\eta_{1}=C=13, \eta_{2}=1$.

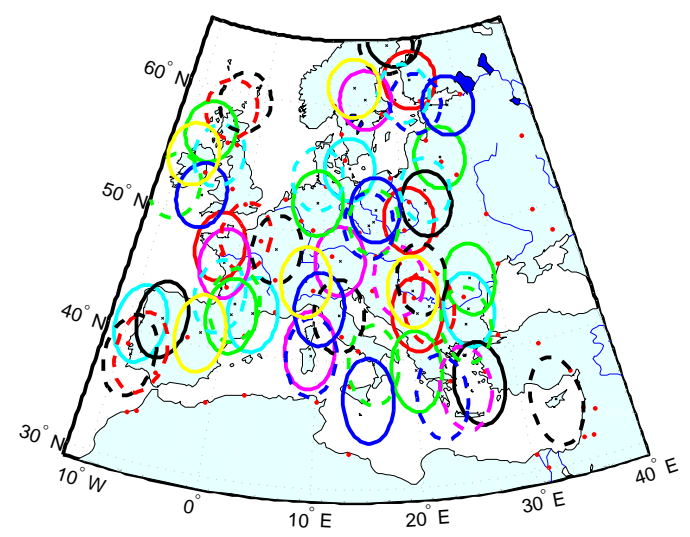

(b) $\eta_{1}=C=13, \eta_{2}=2$.

Fig. 7. Exemplary results of the LDACS FL cell planning. These results demonstrate the feasibility of pan-European LDACS cell planning. Cell size $R_{L D A C S}=120 \mathrm{NMi}, h_{g}=0.01, h_{\min }=0.3048, h_{\max }=18.288$, $\zeta=10 \mathrm{~dB}, d_{c o}=d_{h}^{(L)}$ (worse case). LDACS FL transmit frequencies are \{1048.5: $1: 1060.5\} \mathrm{MHz}$. Channel assignments are encoded in color and line specifications. $\Delta f_{I, \text { Stop }}=\Delta f_{T, \text { Stop }}=93 \mathrm{MHz}$ (worse case).

- We restrict the candidate (and thus the possible) locations in this paper to the locations of the aeronautical VHF-stations, cf. Appendix B However, there are other possibilities. The locations of the DME transponders and/or airports can also 
be considered as candidate locations for LDACS GS.

\section{A. Update Order and Priority of Locations}

The intra-location update can be seen as a competition between the different LDACS FL channels to be assigned at the same possible location (if desired), whereas the extralocation update can be seen as a competition between different possible locations to act as an allocated location. In both cases, the update order decides the "winners". The random update assumes that all possible LDACS FL channels and all possible locations are of the same priority. While this may be true for the LDACS FL channels, some possible locations are, for some reasons, more important than others and thus they are not of the same priority. This aspect can be integrated in the update process. In the following we introduce few examples:

1) If the possible locations are associated with a priority list: Less prior locations are updated first, the most prior location at the end.

2) If a possible location $i$ where $\sum_{c=1}^{C} \boldsymbol{R}(i, c) \geq 1$ is mandatory: Do intra-location update and exclude this location from the extra-location update but take into account its influence on other neurons during the extralocation update.

3) The final result of the LDACS FL cell planning is highly dependent on the candidate locations. A better choice of candidate locations improves the final results.

\section{CONCLusion}

In this paper, we demonstrated that pan-European LDACS cell planning is possible without disturbing the proper operation of DME devices and without requiring the re-allocation of DME frequencies. This is a crucial result for the future aeronautical communications infrastructure relying on LDACS.

The cell planning method used in this paper takes the published geographic distribution of the DME infrastructure into account. We derived closed-form expressions which determine the necessary spectral, spatial and/or power separation between LDACS ground stations and DME devices such that the proper operation of DME devices is not affected. This has been used in a first step to determine possible locations of LDACS ground stations associated with channel assignments which do not disturb the proper operation of the DME system. In a second step, interference constraints have been defined which minimize the interference within LDACS itself. Channel assignment has been achieved by a graph-based approach where the maximum independent set of the graph represents the solution. This solution has been found by a two-state locally asymptotically stable Hopfield network as dynamical solver.

Our results show that, even though many assumptions have been made in favor of the DME system, pan-European coverage of LDACS is possible without affecting the operation of DME. The lower part of the L-band has been assumed for the LDACS reverse link. In this case, only minimal coordination is needed.

\section{REFERENCES}

[1] B. Kamali, "An overview of VHF civil radio network and the resolution of spectrum depletion," in 2010 Integrated Communications, Navigation, and Surveillance Conference Proceedings, May 2010, pp. F4-1-F4-8.

[2] B. Kumar, S. Kumar Dhurandher, and I. Woungang, "A survey of overlay and underlay paradigms in cognitive radio networks," International Journal of Communication Systems, vol. 31, no. 2, 2018.

[3] Final acts WRC-07. Geneva: International Telecommunication Union, 2007. [Online]. Available: http://www.itu.int/dms_pub/itu-s/oth/02/01/ S020100002C4006PDFE.PDF

[4] Final acts $W R C-12$. Geneva: International Telecommunication Union, 2012. [Online]. Available: http://www.itu.int/dms_pub/itu-s/oth/02/01/ s020100003e4002pdfe.pdf

[5] Single european sky joint undertaking. SESAR JU. [Online]. Available: http://www.sesarju.eu/

[6] Next generation air transportation system. Federal Aviation Administration. [Online]. Available: https://www.faa.gov/nextgen/

[7] "Future Communications Infrastructure-Step2: Technology Assessment Results," https://www.eurocontrol.int/sites/default/files/field_tabs/ content/documents/communications/102007-fci-step-2-report-v10.pdf EUROCONTROL, Tech. Rep., October 2007, edition 1.0, Chapter 14.

[8] "Updated LDACS1 system specification," http://www.ldacs.com/wpcontent/uploads/2014/02/LDACS1-Updated-Specification-Proposal-D2Deliverable.pdf Tech. Rep., April 2011.

[9] T. Gräupl and M. Ehammer, "LDACS1 data link layer evolution for ATN/IPS," in Digital Avionics Systems Conference (DASC), 2011 IEEE/AIAA 30th. IEEE, 2011, pp. 4C4-1.

[10] M. Schnell, U. Epple, D. Shutin, and N. Schneckenburger, "LDACS: future aeronautical communications for air-traffic management," IEEE Communications Magazine, vol. 52, no. 5, pp. 104-110, May 2014.

[11] S. Gligorevic, U. Epple, and M. Schnell, "The LDACS1 physical layer design," in Future Aeronautical Communications, S. Plass, Ed. InTech September 2011, ch. 15, pp. 317-332.

[12] U. Epple and M. Schnell, "Overview of interference situation and mitigation techniques for LDACS1," in 30 Digital Avionics Systems Conference, Seattle, WA, October 2011.

[13] _ "Modeling DME interference impact on LDACS1," in Integrated Communications, Navigation and Surveillance Conference, Herndon, VA, April 2012.

[14] X. Hong, C.-X. Wang, H.-h. Chen, and Y. Zhang, "Secondary spectrum access networks," IEEE vehicular technology magazine, vol. 4, no. 2 2009.

[15] M. El Tanab and W. Hamouda, "Resource allocation for underlay cognitive radio networks: A survey," IEEE Communications Surveys \& Tutorials, vol. 19, no. 2, pp. 1249-1276, 2017.

[16] M. G. Khoshkholgh, K. Navaie, and H. Yanikomeroglu, "Access strategies for spectrum sharing in fading environment: overlay, underlay, and mixed," IEEE Transactions on Mobile Computing, vol. 9, no. 12, pp. 1780-1793, 2010.

[17] X. Hong, Z. Chen, C.-X. Wang, S. A. Vorobyov, and J. S. Thompson, "Cognitive radio networks," IEEE vehicular technology magazine, vol. 4, no. 4, 2009.

[18] B. Haindl, J. Meser, M. Sajatovic, S. Müller, H. Arthaber, T. Faseth, and M. Zaisberger, "LDACS1 conformance and compatibility assessment," in 33rd Digital Avionics System Conference, Colorado Springs, CO, 5-9 October 2014.

[19] A. Bertoni, P. Campadelli, and G. Grossi, "A neural algorithm for the maximum clique problem: Analysis, experiments, and circuit implementation," Algorithmica, vol. 33, pp. 71-88, 2002.

[20] V. Lozin, J. Monnot, and B. Ries, "On the maximum independent set problem in subclasses of subcubic graphs," Journal of Discrete Algorithms, vol. 31, pp. 104-112, March 2015.

[21] O. M. Berger, "Neural channel assignment-the fast way," in IEEE International Conference on Neural Networks, vol. 4, Perth, WA, 27 Nov - 01 Dec 1995, pp. 1557 - 1560.

[22] E. Jalali, I. A. Balapuwaduge, F. Y. Li, and V. Pla, "A dynamic channel access strategy for underlay cognitive radio networks: Markov modelling and performance evaluation," Transactions on Emerging Telecommunications Technologies, vol. 28, no. 1, 2017.

[23] J. D. Parsons, The mobile radio propagation channel. John Wiley \& Sons, 2000.

[24] G. Cai, B. M. Chen, and T. H. Lee, "Coordinate systems and transformations," in Unmanned Rotocraft Systems, ser. Advances in Industrial Control. $\quad$ Springer-Verlag London Limited, 2011, ch. 2, pp. 23-34. 
[25] ITU-R, "Technical characteristics of, and protection criteria for nonICAO aeronautical radionavigation systems, operating around $1 \mathrm{GHz}$,' Radio Communications Sector of International Telecommunications Union, Tech. Rep., January 2012, recommendation ITU-R M.2013.

[26] “ICAO Annex 10," Ammendment, Tech. Rep., 1987.

[27] T. L. Singal, "Principles of cellular communication," in Wireless Com munications. Tata McGraw-Hill Education, 2010, ch. 4, pp. 99-127.

[28] R. M. Whitaker and S. Hurley, "Omni-directional cell planning," in Telecommunications Network Design and Management, G. Anandalingam and S. Raghavan, Eds. Kluwer Academic Publisher Boston/Dordrecht/London, 2003, ch. 2, pp. 25-46.

[29] J. J. Hopfield, "Neural networks and physical systems with emergent collective computational abilities," in proceedings of the national academy of sciences of the USA, vol. 79, no. 8, April 1982, pp. 2554-2558.

[30] S. Haykin, Neural networks-A comprehnsive foundation. New York/USA: Macmillan college publishing company, 1994, ch. 8,14.

[31] D. Tank and J. J. Hopfield, "Simple "neural" optimization network: An A/D converter, signal decision circuit, and a linear programming circuit,' IEEE Transactions on Circuits and Systems, vol. 33, no. 5, pp. 553-541, May 1986.

[32] J. J. Hopfield and D. W. Tank, "”Neural" computation of decisions in optimization problems," Biological Cybernatics, vol. 52, no. 3, pp. 141$152,1985$.

[33] A. Engelhart, Vector detection techniques with moderate complexity. VDI Verlag GmbH, 2003, ch. 4, pp. 49-59, dissertation, Ulm University, Institute of Information Technology.

[34] E. Goles and S. Martinez, Neural and automata networks. Dordrecht/Netherlands: Kluwer Academic Publishers, 1990, p. 77.

[35] _ _ "A short proof on the cyclic behavior of multithreshold symmetric automata," Information and Control, vol. 51, no. 2, pp. 95-97, November 1981.

\section{APPENDIX A \\ CONSIDERED DME INFRASTRUCTURE}

Information about the DME infrastructure is periodically published by ICAO in the so-called COM3-Table. We consider all stationary DME and TACAN ground stations listed in the COM3-Table published by ICAO in July 2016. In case of double allocation of the same channel at the same location, we consider that allocation with the larger designated service range. By this we get 2371 relevant DME/TACAN allocations. It is worth mentioning that not all DME/TACAN stations in the COM3-Table are operational ones. Thus, our analysis represents a worse case scenario with respect to the number of DME/TACAN stations.

\section{APPENDIX B}

\section{CONSIDERED AERonAUtiCAL VHF INFrastructure}

Information about the aeronautical VHF communications infrastructure is published periodically by ICAO in the socalled COM2-Table. We consider a subset of non-uniformly distributed stations published in July 2016.

\section{APPENDIX C \\ MaXimum Squared Distance Ratio}

Lemma 1. For a triangle with fixed two vertices $a$ and $b$, and a movable vertex $c$ with a fixed height $h$ as illustrated in Fig. 8 we define

$$
\phi(x)=\frac{d_{2}^{2}(x)}{d_{1}^{2}(x)}>0 .
$$

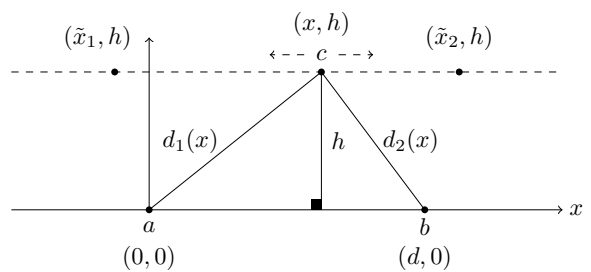

Fig. 8. A triangle with a fixed height $h$, fixed vertices $a$ and $b$ and a movable vertex $c$.

In this case, defining $\alpha$ such that $\sin (\alpha)=\frac{1}{\sqrt{1+\frac{4 h^{2}}{d^{2}}}}$, we get

$$
\begin{aligned}
\phi_{\max }^{(x)} & =\max _{x} \phi(x)=\frac{1+\sin (\alpha)}{1-\sin (\alpha)} \\
\phi(x) & =\phi_{\max }^{(x)} \Rightarrow x=\tilde{x}_{1}=\frac{d}{2} \cdot[1-\csc (\alpha)] .
\end{aligned}
$$

$\csc (\cdot)$ represents the cosecant trigonometric function. Moreover,

$$
\begin{aligned}
& \phi_{\text {min }}^{(x)}=\min _{x} \phi(x)=\frac{1}{\phi_{\text {max }}^{(x)}} \\
& \phi(x)=\phi_{\text {min }}^{(x)} \Rightarrow x=\tilde{x}_{2}=\frac{d}{2} \cdot[1+\csc (\alpha)] .
\end{aligned}
$$

Proof: The situation in Fig. 8 is comparable to Fig. 1 , where the vertex $c$ moves parallel to and directly over the $x$-axes with constant height $h$. From Fig. 8 we notice

$$
\begin{aligned}
\phi(x) & =\frac{(x-d)^{2}+h^{2}}{x^{2}+h^{2}}, \\
\frac{\mathrm{d} \phi(x)}{\mathrm{d} x} & =2 \cdot d \cdot \frac{x^{2}-d \cdot x-h^{2}}{\left(x^{2}+h^{2}\right)^{2}} .
\end{aligned}
$$

$\frac{\mathrm{d} \phi(x)}{\mathrm{d} x}=0 \Rightarrow x=\tilde{x}_{1}$ or $x=\tilde{x}_{2}$. By substituting $\tilde{x}_{1}$ and $\tilde{x}_{2}$ in Eq. 21a and using basic properties of trigonometric functions we obtain

$$
\begin{aligned}
& x=\tilde{x}_{1} \Rightarrow \phi(x)=\phi_{\max }^{(x)}, \\
& x=\tilde{x}_{2} \Rightarrow \phi(x)=\phi_{\text {min }}^{(x)} .
\end{aligned}
$$

We notice also that

- $\lim _{x \rightarrow \pm \infty} \phi(x)=1$.

- $\phi_{\max }^{(x)}>1$.

- $0<\phi_{\min }^{(x)}<1$.

- $\tilde{x}_{1}<0$ (left to $a$ ).

- $\tilde{x}_{2}>d$ (right to $b$ ).

These properties are shown in Fig. 9,10 .

Remark 11. $\forall x \in\left(\tilde{x}_{1}, \tilde{x}_{2}\right): \phi(x)$ is monotonically decreasing.

Lemma 2. $\forall|\epsilon| \neq 0: \phi_{\min }^{(x)}<\frac{d_{2}^{2}(x)+|\epsilon|}{d_{1}^{2}(x)+|\epsilon|}<\phi_{\max }^{(x)}$.

Proof: Based on Lemma 1

- $\phi_{\min }^{(x)} \leq \frac{d_{2}^{2}(x)}{d_{1}^{2}(x)} \leq \phi_{\max }^{(x)}$

- $1-\phi_{\max }^{(x)}<0$

In order to fulfill $\frac{d_{2}^{2}(x)+|\epsilon|}{d_{1}^{2}(x)+|\epsilon|}<\phi_{\max }^{(x)}:|\epsilon|$ must fulfill $|\epsilon|>\frac{d_{1}^{2}(x) \cdot \phi_{\max }^{(x)}-d_{2}^{2}(x)}{1-\phi_{\max }^{(x)}}$. This is always fulfilled $\forall|\epsilon| \neq 0$ since 


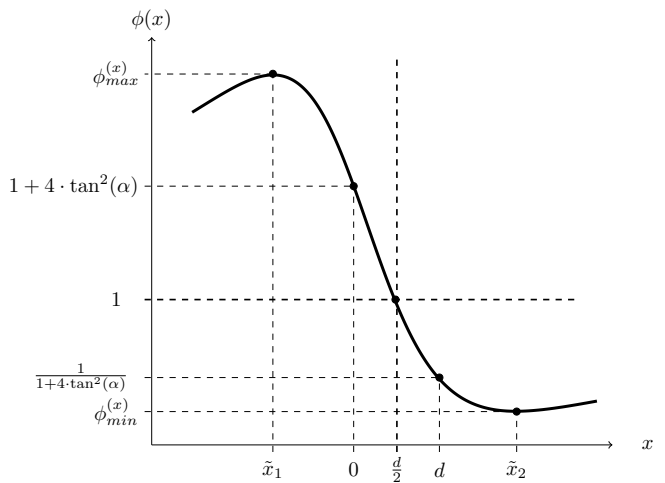

Fig. 9. Illustration of the function $\phi(x)$ in Eq. 18 based on Fig. 8

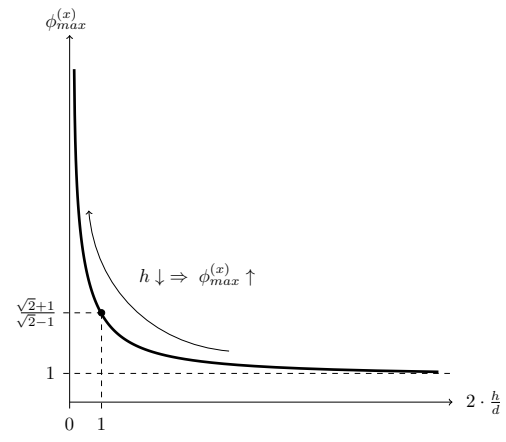

Fig. 10. Illustration of the function $\phi_{\max }^{(x)}$ in Eq. 19a).

the right hand side of this inequality is always non-positive. $\phi_{\min }^{(x)}<\frac{d_{2}^{2}(x)+|\epsilon|}{d_{1}^{2}(x)+|\epsilon|}$ can be proved similarly.

Lemma 3. For a triangle in a three dimensional space with fixed two vertices $a$ and $b$, and a movable vertex $c$ at fixed height $z=h$ as in Fig. 11 we define

$$
\phi(x, y)=\frac{d_{2}^{2}(x, y)}{d_{1}^{2}(x, y)}>0 .
$$

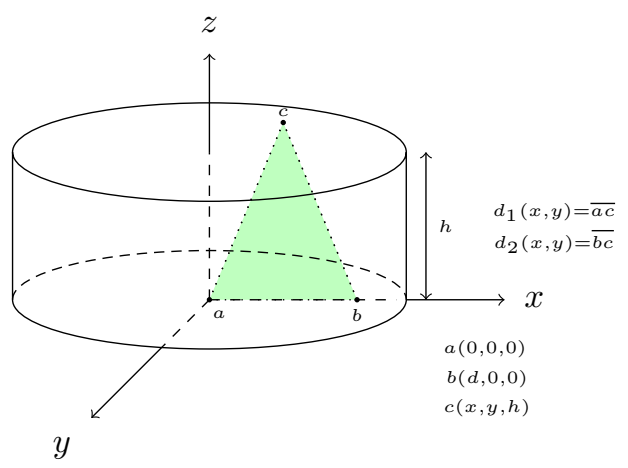

Fig. 11. A triangle in a 3-dimensional space with fixed two vertices $a$ and $b$, and a movable vertex $c$ at fixed height $z=h$.

In this case, it holds

$$
\phi_{\min }^{(x)} \leq \phi(x, y) \leq \phi_{\max }^{(x)}
$$

where $\phi_{\min }^{(x)}$ and $\phi_{\max }^{(x)}$ are given in Eq. 19a,, $20 \mathrm{a}$.
Proof: From Fig. 11 we notice

$$
\begin{aligned}
\phi(x, y) & =\frac{(x-d)^{2}+h^{2}+y^{2}}{x^{2}+h^{2}+y^{2}} \\
& =\frac{d_{2}^{2}(x)+y^{2}}{d_{1}^{2}(x)+y^{2}} .
\end{aligned}
$$

In the last relation $d_{1}(x)$ and $d_{2}(x)$ are according to Lemma 1 Based on Lemma 2 considering $y^{2}=|\epsilon|$

$$
\phi_{\min }^{(x)} \leq \phi(x, y) \leq \phi_{\max }^{(x)} .
$$

The equality in Eq. 26 counts for the case where $y=0$, which reduces $\phi(x, y)$ in Eq. (23) to $\phi(x)$ in Eq. (18).

Remark 12. If the vertex $c$ in Fig. 11 changes its height within a range $h \in\left[h_{\min }, h_{\max }\right]$ as well, then $\phi_{\max }^{(x)}$ and $\phi_{\min }^{(x)}$ are determined by $h_{\min }$ since $\phi_{\max }^{(x)}$ in Eq. (19a) is inversely proportional with $h$, cf. Fig. 10 , and thus $\phi_{\min }^{(x)}=\frac{1}{\phi_{m a x}^{(x)}}$ in Eq. (20a) is proportional with $h$.

\section{We conclude:}

For a triangle in a 3-dimensional space with fixed two vertices $a(0,0,0)$ and $b(d, 0,0)$, and a movable vertex $c(x, y, z)$ with a height $z$ within a range $\left[h_{\min }, h_{\max }\right]$ we define

$$
\phi(x, y, z)=\frac{d_{2}^{2}(x, y, z)}{d_{1}^{2}(x, y, z)}>0 .
$$

given that

$$
\begin{gathered}
d_{2}^{2}(x, y, z)=(x-d)^{2}+y^{2}+z^{2}, \\
d_{1}^{2}(x, y, z)=x^{2}+y^{2}+z^{2}, \\
z \in\left[h_{\min }, h_{\max }\right] . \\
\phi_{\max }=\max _{x, y, z} \phi(x, y, z)=\phi\left(\breve{x}_{1}, 0, h_{\min }\right) \\
=\frac{1+\sin \left(\alpha_{\max }\right)}{1-\sin \left(\alpha_{\max }\right)}, \\
\breve{x}_{1}=\frac{d}{2} \cdot\left[1-\csc \left(\alpha_{\max }\right)\right]<0 . \\
\phi_{\min }=\min _{x, y, z} \phi(x, y, z)=\phi\left(\breve{x}_{2}, 0, h_{\min }\right) \\
=\frac{1-\sin \left(\alpha_{\max }\right)}{1+\sin \left(\alpha_{\max }\right)}, \\
\breve{x}_{2}=\frac{d}{2} \cdot\left[1+\csc \left(\alpha_{\max }\right)\right]>d .
\end{gathered}
$$

where

$$
\cot \left(\alpha_{\max }\right)=2 \cdot \frac{h_{\min }}{d} .
$$

We notice that $\breve{x}_{1}$ lies on that side of $a$ which is far away from $b$. In addition $d-\breve{x}_{1}=\breve{x}_{2}$. The smaller $h_{m i n}$, the closer is $\breve{x}_{1}$ to $a$ and $\breve{x}_{2}$ to $b$.

Remark 13. In a special case where the vertices $a$ and $b$ coincide (co-site case), i.e. $d=0(\alpha=0)$, it holds $\forall x, y, z: \phi(x, y, z)=1$.

Remark 14. If in Fig. $11 x \in\left[\frac{d}{2}, \infty\right)$, then based on Eq. 27], (28) $\phi(x, y, z) \leq 1 \forall y$, z, i.e. for this case $\phi_{\max }=1$. This is also shown in Fig. 9 
APPENDIX D

ACRONYMS AND ABBREVIATIONS

AM(R)S Aeronautical Mobile (Route) Service

ARNS Aeronautical Radio Navigation Service

ATM Air Traffic Management

DME Distance Measuring Equipment

DOC Designated Operation Conditions

ECEF Earth-Centered Earth-Fixed Coordinate System

FAA Federal Aviation Administration

FCI Future Communications Infrastructure

FL Forward Link

FL $x \quad$ Flight Level (FL $x=100 \cdot x \mathrm{ft}$ above ground)

GPS Global Positioning System

GS Ground Station

ICAO International Civil Aviation Organization

LDACS L-band Digital Aeronautical Communications System

NextGen Next Generation Air Transportation System

NP Nondeterministic Polynomial time

OFDM Orthogonal Frequency-Division Multiplexing

RL Reverse Link

SESAR Single European Sky ATM Research

TACAN Tactical Air Navigation

WGS84 World Geodetic System 1984 